\title{
Synthesis and 3D-AFM surface topology of nanographene-like material extracted from sulfonated tri-, di- and monochloroacetic acid
}

\author{
KHALED M. ELSABAWY ${ }^{1,2, *}$ \\ ${ }^{1}$ Chemistry Department, Faculty of Science, Tanta University, Tanta-31725, Egypt \\ ${ }^{2}$ Chemistry Department, Faculty of Science, Materials Science Unit, Taif University, \\ 888-Alhawya, Taif City, Kingdom of Saudi Arabia
}

\begin{abstract}
In this study a graphene-like material was synthesized by chemical exfoliation of sieved graphite powder with an average particles size $\leqslant 100 \mu \mathrm{m}$ in dispersing medium from tri-, di- and monochloroacetic acid sulfonated with $55 \% \mathrm{H}_{2} \mathrm{SO}_{3}$. The results indicated that the yield of graphene-like material was the best in case of trichloroacetic $55 \% \mathrm{H}_{2} \mathrm{SO}_{3}$, taking into account structure quality and amount of obtained material. Layered graphene-like material was carefully collected after reduction of GO formed by $50 \%$ dimethylhydrazine. AFM-investigations were performed to characterize nanostructural features of produced graphene and expected surface area which is surface topology dependent. Furthermore, Raman spectra were measured to confirm graphene formation.
\end{abstract}

Keywords: synthesis; dispersion; AFM; graphene; Raman spectra

(C) Wroclaw University of Technology.

\section{Introduction}

It is well known that graphene is a flat monolayer of carbon atoms tightly packed into a two-dimensional honeycomb lattice, forming the basic building block for all carbon nanostructures [1]. Graphene showns many intriguing properties which makes it one of the most promising materials in the next decades [2,3].

In 2004, Novoselov et al. [4] developed a micromechanical exfoliation method that consisted of repeated peeling of graphite flakes, using adhesive tape until the thinnest flakes were obtained, which were then transferred onto a clean substrate by gentle pressing of the tape. This method relies on the balance between the interlayer cohesion and long distance interactions between the tape or the substrate and graphene [1].

In the chemical exfoliation process, the introduction of reactants in the interlayer space weakens the van der Waals cohesive force. The

*E-mail: khaledelsbawy@yahoo.com loosened layer stacking is disrupted when the intercalant decomposition produces a high gas pressure of $\mathrm{CO}_{2}$ by a rapid annealing to $1050{ }^{\circ} \mathrm{C}$. As a result, the sp2 lattice is partially degraded to sp2-sp3 sheet that possesses less $\pi-\pi$ stacking stability.

Chemical exfoliation can be performed in a suspension known as graphite oxide. The most common method to produce graphite oxide was reported by Hummers et al. [5], where graphite was dispersed in a mixture of concentrated sulfuric acid, sodium nitrate and potassium permanganate at $45{ }^{\circ} \mathrm{C}$ for a couple of hours. In that compound, the graphite layers remained largely intact and the quest molecules or atoms were located in between. To obtain a few or even single sheet materials, the intercalated reactant was decomposed to produce large amounts of gas in the van der Waals space by chemical or thermal means [6]. Then, rapid annealing to $1050{ }^{\circ} \mathrm{C}$ generated a $\mathrm{CO}_{2}$ overpressure and split the graphite oxide into individual sheets [7]. Graphene oxide solutions were yellow in color or greenish-blue when non-oxidized graphene sheets were the major constituents $[8,9]$. 
GO can be regarded as graphene functionalized by carboxylic acid, hydroxyl and epoxide groups. Hence, these functionalized groups make GO easily dispersed in a few selected polar solvents that form an intercalated composite with polar molecules $[10,11]$. The intercalation of solvent causes the graphene sheet to swell and lose its mechanical integrity [12]. Nevertheless, large quantities of structural defects introduced by the oxidation process shift the physical properties away from pristine graphene [13].

It is well known that flake graphite and exfoliated (expandable) graphite are generally used to prepare graphite oxide. Several methods for the preparation of graphite oxide have been reported [14-18]. Hummers' original method has been modified in several ways to increase the degree of oxidation of graphite. Pre-oxidized graphite has also been used to prepare graphite oxide by the modified Hummers' method [15]. The Staudenmaier method is also an effective way to produce chemically oxidized graphite. A few other oxidants such as Jones' reagent $\left(\mathrm{H}_{2} \mathrm{CrO}_{4} / \mathrm{H}_{2} \mathrm{SO}_{4}\right)$, Brodie's reagent, and peroxides have been used to oxidize graphite [14, 17, 18].

The major goal of the present work is to introduce a new simple dispersed matrix for exfoliation of GO to yield graphene-like material with a reasonable structural quality.

\section{Experimental}

\subsection{Sample preparation}

Graphene-like material was prepared according to the following sequence of steps: $3 \mathrm{mg}$ of sieved graphite powder with particle size $\leqslant 100 \mu \mathrm{m}$ (Merck product) was dispersed in $25 \mathrm{~mL}$ of trichloroacetic acid $55 \% \mathrm{H}_{2} \mathrm{SO}_{3}$ by bath sonication. The sonication of the dispersion process was performed in an ultrasonic bath (WiseClean WUCDIOH $200 \mathrm{~W}, 40 \mathrm{kHz}$ ) up to 60 hours at two stages of mechanical stirring: first after $5 \mathrm{~h}$ and second after $20 \mathrm{~h}$, each of $1 \mathrm{~h}$ duration. Dispersion by bath sonication provided the mechanical disruption that broke apart the graphite flakes, which were then stabilized by the solvent system.
After the sonication process, the dispersion was dark grey in color as shown in Fig. 1a, and left to stand for about $10 \mathrm{~h}$ to allow small aggregates to form.

To remove these aggregates and stabilize the dispersion, the top $15 \mathrm{~mL}$ of the dispersion was taken out and consequently centrifuged for $40 \mathrm{~min}$ utes at $\sim 10,000 \mathrm{rpm}$. After this primary centrifugation, the top $10 \mathrm{~mL}$ of the dispersion was decanted carefully by pipette, forming a homogeneous black dispersion which was retained for use.

These procedures were identically repeated for the other two solvents, namely: di- and monochloroacetic acid $\begin{array}{llllll}55 & \mathrm{H}_{2} \mathrm{SO}_{3} & \text { (Fig. } 1 \mathrm{~b}\end{array}$ and Fig. 1c).
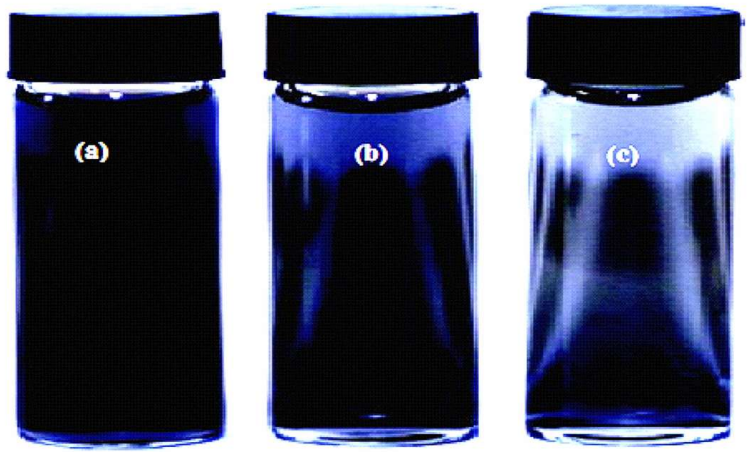

Fig. 1. Gray color gradient for graphite dispersed in different substituted chloroacetic acids: (a) trichloroacetic acid, (b) dichloroacetic acid, (c) monochloroacetic acid.

No sedimentations were noticed for the first 10 hours which confirms that the graphene oxide (GO) was relatively stable in the choloroacetic in contrast to some other organic solvents. It is well known that water is recognized as a medium for self-assembly of GO. Jalili et al. [19] studied dispersed ultra-large GO sheets in a number of common solvents including water, $\mathrm{N}-\mathrm{N}$-dimethylformamide (DMF), N-cyclohexyl-2pyrrolidone (CHP), tetrahydrofuran (THF), acetone, ethanol and a number of other organic solvents, many of which were not known to afford solvophobic self-assembly before. All of the non-polar solvents were ineffective in dispersing GO and therefore could not create any LC phase 
as expected. Hence, in our report we tried to introduce a new simply dispersed solvent which is trichloroacetic acid, liable to apply in easy way, with a moderate to high yield and good structure quality.

Only a few solvents other than water are capable of supporting amphiphilc self-organization [20, 21]. These solvents include a very limited range of multifunctional alcohols (such as ethylene glycol), amides and a wide range of protic ionic liquids widely known as PILs [22, 23].

Finally, $13 \mathrm{~mL}$ of dimethyl-hydrazine solution (50\%) $\left(\mathrm{CH}_{3} \mathrm{NH}_{2}-\mathrm{NHCH}_{3}\right)$ was added carefully dropwise to get graphene-like material in highly pure state, leaving the solution settled for $5 \mathrm{~h}$. Then the solution was centrifuged in order to dispose the thickest flakes of nanographene-like material as possible.

\subsection{Structural measurements}

$\mathrm{X}$-ray diffraction (XRD) measurements were carried out at room temperature on fine ground samples, using $\mathrm{CuK} \alpha$ radiation source, Ni-filter and a computerized STOE diffractometer (Germany) with $2 \theta$ step scan technique. Rietveld refinement and indexing of structure were made via Fullprof package and Gesas program.

\subsection{Raman spectroscopy measurements}

The measurements of Raman spectra were carried out on the fine ground powders at a laser wavelength of $632.8 \mathrm{~nm}$ (He-Ne laser with power of $1 \mathrm{~mW}$ ) and laser power applied to the test site of $0.4 \mathrm{~mW}$, using a microscope objective with $\times 20$ magnification, accumulation time of $1000 \mathrm{~s}$ to $4000 \mathrm{~s}$, in the time exceeding an hour.

\subsection{Atomic force microscopy (AFM)}

High-resolution atomic force microscope (AFM) was used for testing morphological features and topological map (Veeco-di Innova Model-2009-AFM-USA). The measurements were performed in tapping non-contacting mode. For accurate mapping of the surface topology AFM raw data were forwarded to the Origin-Lab version 6-USA program to visualize more accurately three dimensional surface of the sample under investigation. This process is a new trend to get high resolution 3D-mapped surface [24-26].

\section{Results and discussion}

The synthesized graphene-like material flakes were investigated by both Raman spectroscopy and $\mathrm{X}$-ray diffraction to prove the existence of graphene as shown in Fig. 2a and Fig. 2b. As it is seen in Fig. 2a, characteristic graphene peaks (modes) appear in the wavelength range of $1400 \mathrm{~cm}^{-1}$ to $1650 \mathrm{~cm}^{-1}$ which is fully consistent with Elsabawy [27], while graphene oxide appears at $\sim 1000 \mathrm{~cm}^{-1}$ and $\sim 1600 \mathrm{~cm}^{-1}$ as reported in the literature [28-30].

Saito et al. [28] reported that in graphene, the Stokes phonon energy shift caused by laser excitation creates two main peaks in the Raman spectrum: $\mathrm{G}\left(1580 \mathrm{~cm}^{-1}\right)$, a primary in-plane vibrational mode, and 2D $\left(2690 \mathrm{~cm}^{-1}\right)$, a secondorder overtone of different in-plane vibration, D $\left(1350 \mathrm{~cm}^{-1}\right)$. D and 2D peak positions are dispersive (dependent on the laser excitation energy) [29]. Furthermore, the D peak of GO located at $1352 \mathrm{~cm}^{-1}$ and at $1350 \mathrm{~cm}^{-1}$ for rGO steams from a defect-induced breathing mode of sp2 rings [30]. It is common for all sp2 carbon lattices and arises from the stretching of $\mathrm{C}-\mathrm{C}$ bond. The $G$ peak at around $1600 \mathrm{~cm}^{-1}$ for GO and at $1599 \mathrm{~cm}^{-1}$ for rGO is due to the first order scattering of the E2g phonon of sp2 C atoms [30].

From Fig. 2a one can see that the original experimental Raman profile is very crowded and there are many Raman modes overlapping with each other due to GO existence, as can be seen in the refined smoothed Raman profile.

Although XRD measurement is not an accurate tool to identify graphene-like material but in our case as it is clear in high resolution XRD profile, it gives sharp peak at $2 \theta \sim 24.99^{\circ}$ which is fully consistent with literature [31]. The shift in the fingerprint peak position of graphene-like material is attributable to interference of polycrystalline phases that exist together with graphene-like material, such as graphene oxide and small traces of unreacted graphite. 


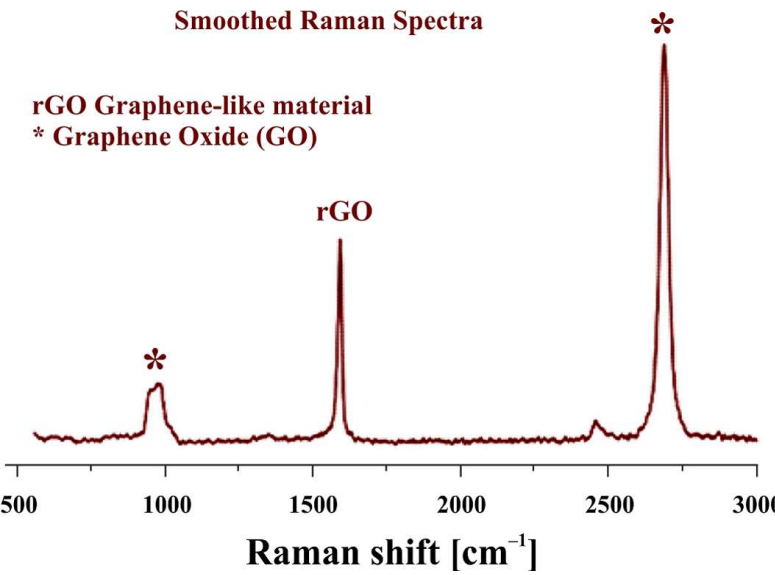

(a)

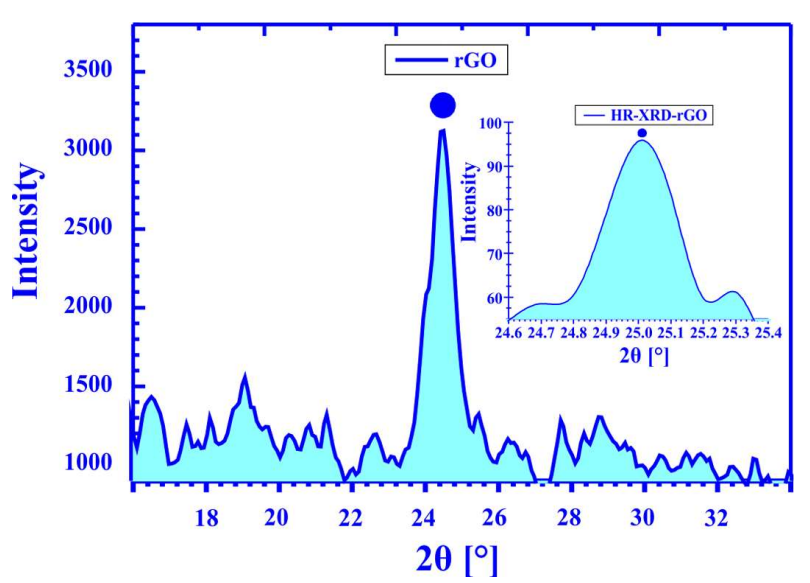

(b)

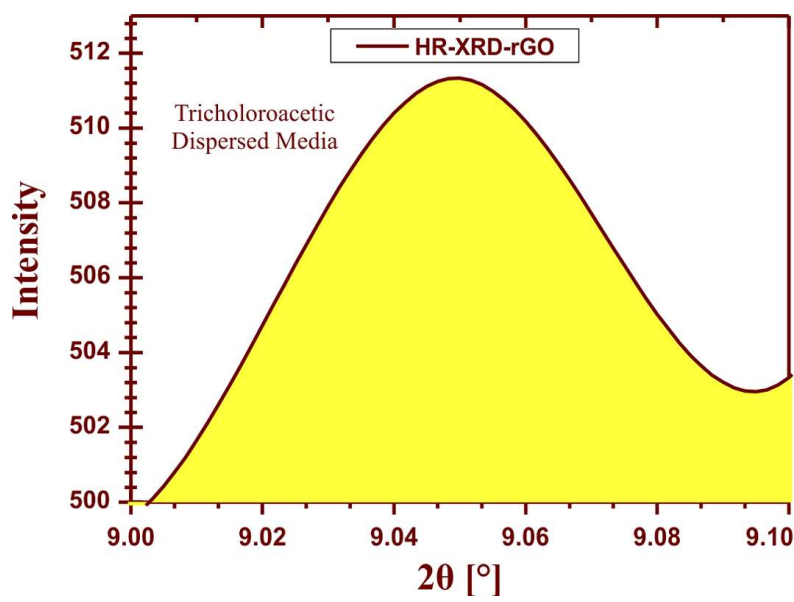

(c)

Fig. 2. (a) Raman spectrum measured for ground graphene flakes, (b) X-ray diffraction pattern recorded for graphene-like material flakes, (c) high-resolution XRD pattern at very narrow $2 \theta$ range recorded for graphene-like material dispersed in tricholoroacetic acid as a solvent.
Fig. 2c displays HR-XRD-pattern recorded for graphene-like material applying tricholoroacetic acid as dispersing solvent. There is a medium broad peak at $2 \theta$ value $\sim 9.045^{\circ}$ which matches with the results by Jalili et al. [19] who investigated the effect of six different kinds of solvents including water, acetone, DMF and THF on the GO formation. They recorded specific peak appearing at $2 \theta$ range of $8^{\circ}$ to $11^{\circ}$ attributable to individual GO platelets interlinked via a non-uniform network of hydrogen bonds mediated by oxygenated functional groups and solvent molecules.

To characterize nanostructural features of obtained graphene-like material AFM measurements were carried out in tapping non-contact mode as shown in Fig. 3.

Fig. 3 shows 2D- and 3D-AFM images captured for tiny scanned area $\left(0.1 \times 0.1 \mu^{2}\right)$ of graphenelike material. Fig. 3c displays honeycomb ultrastructure of graphene-like material which was synthesized via CVD and reported in the literature [27] by author himself applying STM atomic mode.

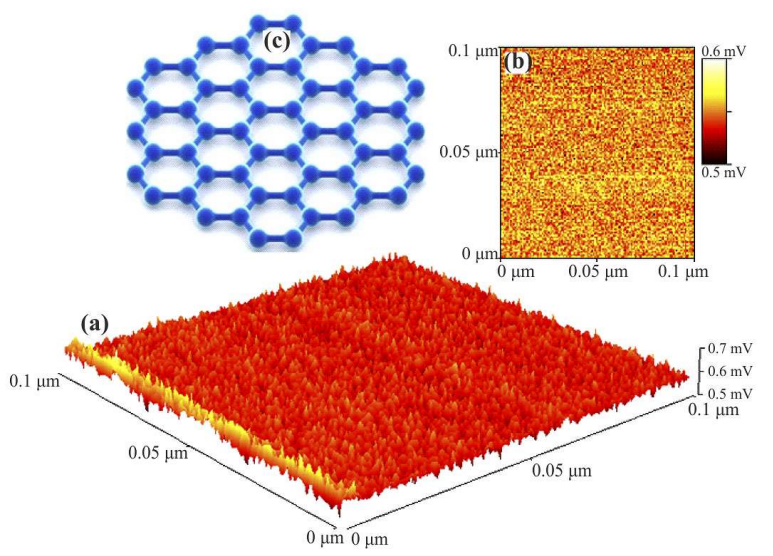

Fig. 3. (a) 3D-image for graphene-like material in tapping non-contact mode, (b) 2D-AFM image for graphene-like material in tapping non-contact mode, (c) honeycomb structure of graphene.

For accurate mapping of surface topology, AFM-raw data were forwarded to the OriginLab version 6-USA program to visualize more accurately three dimensional surface of the synthesized nanographene. Fig. 4 represents a very narrow 3D-scanned area with the size of 


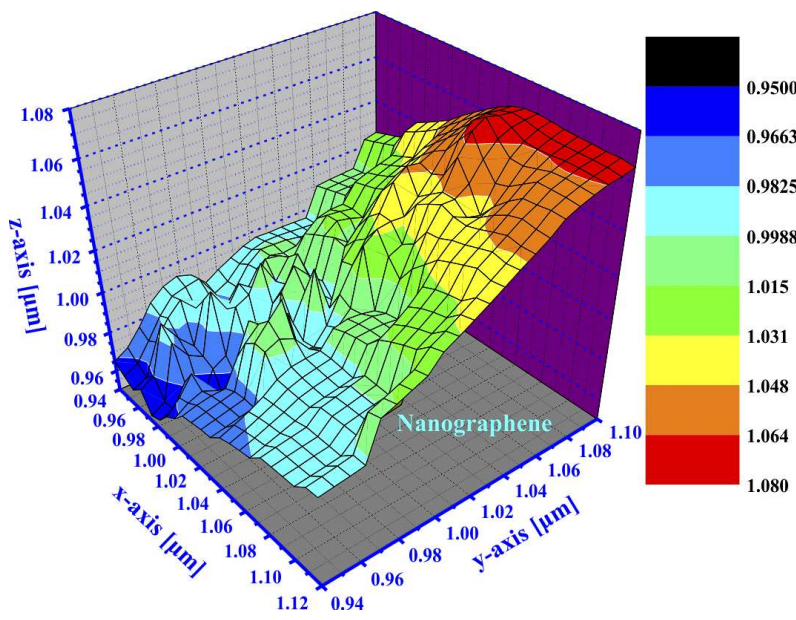

Fig. 4. 3D-AFM-visualized image for the synthesized graphene-like material.

$0.16 \mu \mathrm{m} \times 0.16 \mu \mathrm{m} \times 0.16 \mu \mathrm{m}$. These accurate investigations explain why graphene has so unique huge exposure surface area $\left(\sim 2600 \mathrm{~m}^{2} / \mathrm{g}\right)$, as reported in the literature $[32,33]$, with different gradients on the surface topology in contrast to other carbon-based materials.

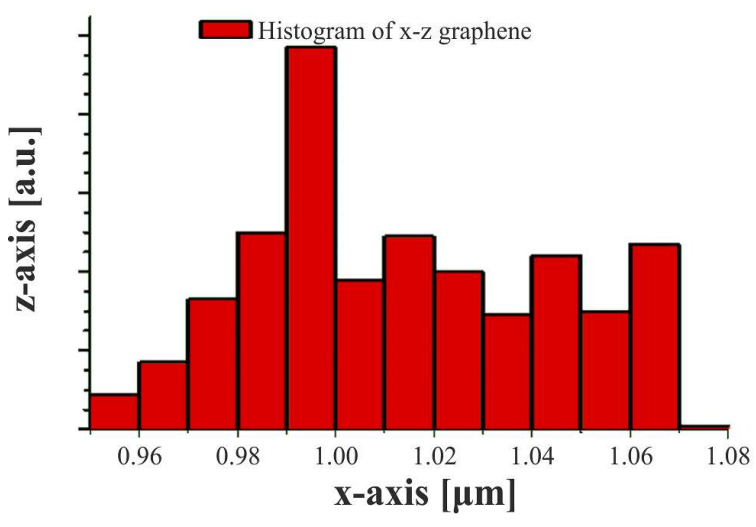

Fig. 5. Histogram of $x-z$ axes of graphene-like material.

Fig. 5 displays cross sectional view through $\mathrm{X}-\mathrm{Z}$ axis of graphene-like material, showing height gradient on the surface which qualifies the graphene-like material to play an important role in catalysis and industrial applications.

\section{Conclusions}

Graphene-like material with a reasonable structural quality has successfully been synthesized by exfoliation in tri-, di- and monochloroacetic acid sulfonated with $55 \% \mathrm{H}_{2} \mathrm{SO}_{3}$ as dispersed matrix. The results indicate that as polarity of dispersing solvent increases (trichloro-, dichloro-, monochloroacetic) the yield of GO increases and consequently the amount of graphene-like material (rGO) increases. Fingerprint peak position of the graphene-like material, which lies at $2 \theta$ value of $\sim 24.8^{\circ}$, has shifted by a small value attributable to polycrystalline phases interference that exist together with graphene-like material as non-reduced graphene oxide and small traces of unreacted graphite. Accurate AFM-mapping of the graphene-like material confirms that the graphenelike material has unique nanofeatures accompanied by a very huge surface area which qualifies it as an active surface catalyst.

\section{References}

[1] Soldano C., Mahmood A., Dujardin E., Carbon, 48 (2010), 2127.

[2] Geim A.K., Novoselov K.S., Nat. Mater, 6 (2007), 183.

[3] PARK S., RuOFF, R.D., Nat. Nanotechnol., 4(2009), 217.

[4] Novoselov K.S., Geim A.K., Morozov S.V., JiAnG D., Zhang Y., Dubonos S.V., Grigorieva I.V., FIRSOV A.A., Science, 306 (2004), 666.

[5] Hummers W.S., Offeman R.E., J. Am. Chem. Soc., 80 (6) (1958), 1339.

[6] Dressehaus M.S., Dressehaus D., Adv. Phys., 51 (1) (2009), 186.

[7] Schniepp H.C., Li J.L., McAllister M.J., SAi H., Herrera-Alonso M., Adamson D.H., J. Phys. Chem. B, 110 (17) (2006), 8535.

[8] Mac A.M.J., Li J.L., Adamson D.H., SchniepP H.C., Abdala A.A., LiU J., Chem. Mater, 19 (18) (2007), 4396.

[9] Li D., Muller M.B. Gilje S., Kaner R.B., WalLACE G.G., Nat. Nanotechnol., 3 (2) (2008), 101.

[10] Chen Y., Qi Y., Tai Z., Yan X., Zhu F., Eur. Polym. J., 48 (2012), 1026.

[11] Zhou X.Z., Huang X., QI X.Y., Wu S.X., Xue C., Boey F.Y.C., Yan Q., Chen P., Zhang H., J. Phys. Chem. C, 113 (2007), 10842.

[12] Stankovich S., Dikin, D.A., Compton O.C., DOMMETT G.H.B., RuOFF R.S., NGUYEN S.T., Chem. Mater., 22 (2010), 4153. 
[13] Hernandez Y., Nicolosi V., Lotya M., Blighe F.M., Sun Z., DE S., Nat. Nanotechnol., 3 (9) (2008), 563.

[14] Shen J., Hu Y., Shi M., Lu X., Li C., Ye M., Chem. Mater., 21 (2009), 3514.

[15] Choi B.G., Park H., Park T.J., Yang M.H., Kim J.S., JANG S.Y., HeO N.S., LEE S.Y., KONG J., HoNG W.H., ACS Nano, 4 (2010), 2910.

[16] Hummers W.S., Offeman R.E., J. Am. Chem. Soc., 80 (1958), 1339.

[17] Zhu Y., Murali S., Ca W.I., Li X., SuK J.W., PotTs J.R., RuOFF R.S., Adv. Mater., 22 (2010), 3906.

[18] Zhang Y., Ren L., Wang S., Marathe A., ChaudHURI J., LI G., J. Mater. Chem., 21 (2011), 5386.

[19] Jalili R., Aboutalebi S., EsRafilzadeh D., Konstantinov K.K., Moulton S.E., Razal J.M., Wallace G.G., ACS Nano, 7 (5) (2013), 3981.

[20] Greaves T.L.; WeErawardena A., Drummond C.J., Phys. Chem. Chem. Phys., 13 (2011), 9180.

[21] Greaves T.L., Drummond C.J., Chem. Soc. Rev., 37 (2008), 1709.

[22] Ray A., J. Am. Chem. Soc., 91(1969), 6511.

[23] Greaves T.L., Weerawardena A., Fong C., DRUMMOND C.J., Langmuir, 23 (2006), 402.
[24] Alhuthali A., El-Nahass M.M., Atta A.A., ElRaheEM A.M.M., Elsabawy K.M., Hassanien A.M., J. Lumin., 158 (2015), 165.

[25] Elsabawy K.M., Int. J. Chem. Concept.,1 (2015), 38.

[26] Elsabawy K.M., World Sci. News, 30 (2016), 57.

[27] ElsabaWy K.M., RSC Adv., 1 (2011), 964.

[28] Saito R., Hofmann M., Dresselhaus G., Jorio A., Dresselhaus M.S., Adv. Phys., 30 (2011), 413.

[29] Ferrari A.C., Solid State Commun., 143 (2007), 47.

[30] Ferrari A.C., Robertson J., Phys. Rev. B, 61 (2000), 14095.

[31] Changuing F., Guogang Z., Haijun Z., Int. J. Electrochem. Sc., 8 (2013), 6269.

[32] Geim A.K., Novoselov K.S., Nat. Mater, 8 (2009), 183.

[33] PARK S., RUOFF R.D., Nat. Nanotechnol., 4 (2009), 217.

Received 2016-01-08 Accepted 2016-06-29 